\title{
Placental protein 14 as a potential biomarker for diagnosis of preterm premature rupture of membranes
}

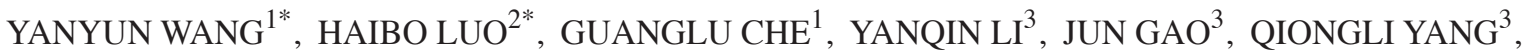 \\ BIN ZHOU ${ }^{1}$, LINBO GAO $^{1}$, TAO WANG ${ }^{1}$, YUJIE LIANG ${ }^{3}$ and LIN ZHANG ${ }^{1}$ \\ ${ }^{1}$ Laboratory of Molecular Translational Medicine, Center for Translational Medicine, \\ Key Laboratory of Birth Defects and Related Diseases of Women and Children, Ministry of Education, \\ Department of Obstetrics and Gynecology, West China Second University Hospital; ${ }^{2}$ Institute of Forensic Medicine, \\ West China School of Basic Sciences and Forensic Medicine, Sichuan University, Chengdu, Sichuan 610041; \\ ${ }^{3}$ Department of Perinatal Healthcare, Shuangliu District Maternal and Child Health Hospital, \\ Chengdu, Sichuan 610200, P.R. China
}

Received January 15, 2018; Accepted April 3, 2018

DOI: $10.3892 / \mathrm{mmr} .2018 .8967$

\begin{abstract}
Premature rupture of membranes (PROM) is a common pregnancy complication that frequently results in maternal and perinatal morbidity. The present methods for diagnosing PROM do not satisfy clinical requirements. The present study aimed to examine the proteome profile of amniotic fluid (AF) and maternal plasma, screen unique proteins in $\mathrm{AF}$, and evaluate their diagnostic value for diagnosing PROM. The proteome profiles of AF and maternal plasma were examined via liquid chromatography coupled with tandem mass spectrometry-based proteomic techniques. The protein expression levels of diagnostic candidates in AF, maternal plasma and vaginal fluid were determined by ELISA analysis and Magnetic Luminex ${ }^{\circledR}$ screening assays. The diagnostic value of potential biomarkers was evaluated using receiver operating characteristic curves. A lateral flow assay was developed based on colloidal gold immunochromatography
\end{abstract}

Correspondence to: Dr Yujie Liang, Department of Perinatal Healthcare, Shuangliu District Maternal and Child Health Hospital, 396 Jian Cao Zhong Jie Road, Chengdu, Sichuan 610200, P.R. China E-mail: 364150666@qq.com

Professor Lin Zhang, Laboratory of Molecular Translational Medicine, Center for Translational Medicine, Key Laboratory of Birth Defects and Related Diseases of Women and Children, Ministry of Education, Department of Obstetrics and Gynecology, West China Second University Hospital, 20 Ren Min Nan Lu Road, Chengdu, Sichuan 610041, P.R. China

E-mail: zhanglin@scu.edu.cn

${ }^{*}$ Contributed equally

Key words: premature rupture of membranes, placental protein 14, biomarker, liquid chromatography-tandem mass spectrometry, lateral flow assay technology. The present study identified 540 unique proteins in AF, 12 of which were chosen for further detection. The present results demonstrated that expression levels of pulmonary surfactant-associated protein B, BPI fold-containing family A member 1, zymogen granule protein 16 homolog B, EGF-containing fibulin-like extracellular matrix protein 1, keratin, type II cytoskeletal 4, keratin, type I cytoskeletal 19, placental protein 14 (PP14), insulin-like growth factor-binding protein 2, mesothelin and serpin family B member 3 were significantly higher in AF compared with in maternal plasma $(\mathrm{P}<0.01)$. Furthermore, PP14 was observed to have excellent diagnostic accuracy for preterm PROM (PPROM), with a respective sensitivity and specificity of 100 and $87.5 \%$ when the cutoff value was $0.008 \mu \mathrm{g} / \mathrm{ml}$. The PP14-based lateral flow assay demonstrated a visual detection threshold of $0.008 \mu \mathrm{g} / \mathrm{ml}$. The results from the present study suggested that PP14 may be a novel potential biomarker for PPROM, and may be developed into a lateral flow assay for bedside application to rapidly diagnose PPROM.

\section{Introduction}

Premature rupture of membranes (PROM) is a common complication during pregnancy (1). According to a previous study, PROM complicates $24.3 \%$ of all pregnancies in Beijing, China (2). PROM, particularly preterm PROM (PPROM), frequently results in maternal and perinatal morbidity. The complications of PROM include premature labor, chorioamnionitis, respiratory distress syndrome, cord compression, placental abruption and antepartum fetal mortality (3). Pregnant women with vaginitis, a history of premature labor or twin pregnancies, have a higher risk of developing PROM (4). The etiology of PROM is unknown and its occurrence is unpredictable (5). Therefore, accurate and timely diagnosis may improve maternal and perinatal outcomes.

In the past 80 years, several techniques to clinically diagnose PROM have been developed, including microscopic fetal cell identification, vaginal fluid $\mathrm{pH}$ determination, examination 
of amniotic fluid (AF) crystallization, intra-amniotic dye injection and protein marker detection in the vaginal fluid. The most accurate method to diagnose PROM is with an intra-amniotic dye injection, using a dye such as indigo carmine. However, this method is invasive and may cause infection or abortion (5). Fetal cell identification or AF crystallization examination with a microscope are time-consuming processes and false positive rates are high. Therefore, vaginal $\mathrm{pH}$ tests and a number of specific PROM marker-based bedside test products are more popular at present due to their safety, efficiency and convenience (3,6-9). However, during clinical practice, patients with suspected PROM frequently experience vaginal bleeding and excessive blood in the cervical-vaginal fluid (VF) that may interfere with rapid tests and result in false positive outcomes, which is concerning for clinicians.

In order to improve the diagnostic accuracy of PROM, the present focused on screening for novel PROM biomarkers. As PROM occurs, AF disseminates into the vagina, and the detection of unique fetal proteins in the VF may aid in the diagnosis of PROM. Therefore, in the present study, the proteome profiles of $\mathrm{AF}$ and maternal plasma of pregnant women was assessed by liquid chromatography coupled with a tandem liquid chromatography-mass spectrometry (LC-MS/MS)-based proteomic technique. Unique proteins in AF were screened and preliminarily evaluated for their potential diagnostic ability in PROM by using ELISA and a Magnetic Lumine ${ }^{\circledR}$ screening assay to determine a potential biomarker for PPROM.

\section{Materials and methods}

Subjects. The present study was approved by the Ethics Committees of West China Second University Hospital of Sichuan University (Sichuan, China) and Shuangliu District Maternal and Child Health Hospital (Sichuan, China). All women ( $\geq 18$ years old) enrolled in the study signed consent forms. In the PPROM/PROM group, the inclusion criteria were defined as follows: Leaking of AF observed prior to labor; $\mathrm{pH}$ test positive; AF crystallization test positive and sICAM-1 strip test positive. For intact membrane group, women who met the following criteria were recruited in the study: No leaking of AF observed prior to labor; $\mathrm{pH}$ test negative; AF crystallization test negative and sICAM-1 strip test negative $(9,10)$. Women who had been administered drugs vaginally in the last $72 \mathrm{~h}$ were excluded from both groups. A total of 133 maternal plasma, $133 \mathrm{AF}$ and 133 VF samples were collected from January 2015 to October 2016. Among them, 100 maternal plasma and $100 \mathrm{AF}$ samples were collected separately from pregnant women in their third trimester, and 33 maternal plasma and $33 \mathrm{AF}$ samples were provided by women who selected amniocentesis in their second trimester. All 133 women were without any complex pregnancy-associated diseases. A total of $71 \mathrm{VF}$ samples were obtained from 14 patients with PPROM ( $<37$ weeks; five patients with vaginal bleeding) and 57 patients with PROM ( $\geq 37$ weeks; 11 patients with vaginal bleeding). The remaining $62 \mathrm{VF}$ samples were collected from pregnant women at $\geq 37$ weeks ( 53 women, five of which experienced vaginal bleeding) or $<37$ weeks (nine women, one of whom experienced vaginal bleeding) with intact membranes.
The clinical characteristics of all subjects are presented in Table I.

Sample collection. Maternal plasma and AF samples were obtained from the women during their third trimester by cesarean section. Samples from women in their second trimester were collected during amniocentesis. A total of $3 \mathrm{ml}$ EDTA-anticoagulated peripheral whole blood and $10 \mathrm{ml}$ uncontaminated AF was collected from each woman. VF samples were collected from the posterior fornix using vaginal swabs. The swabs were inserted in $5 \mathrm{ml}$ microcentrifuge tubes containing $1 \mathrm{ml}$ sterile $0.01 \mathrm{M}$ PBS, and rotated for $30 \mathrm{sec}$. All samples were centrifuged at $400 \mathrm{xg}$ for $10 \mathrm{~min}$ at $4^{\circ} \mathrm{C}$, and the supernatant was removed, aliquoted and stored at $-80^{\circ} \mathrm{C}$.

LC-MS/MS analysis. A total of 10 maternal plasma and $10 \mathrm{AF}$ samples which originated from five second trimester women and 10 third trimester women were separately mixed into a plasma sample and an AF sample pool. Protein identification in the two samples was performed using the LC-MS/MS technique. The whole procedure may be defined as follows. Firstly, the proteins in the two samples were separately enriched and extracted using ProteoMiner ${ }^{\mathrm{TM}}$ kits (Bio-Rad Laboratories, Inc., Hercules, CA, USA) for LC-MS/MS analysis, according to the manufacturer's protocol. Secondly, $100 \mu \mathrm{g}$ total protein/sample was digested into peptides using trypsin with a protein/enzyme ratio of $30: 1(\mathrm{w} / \mathrm{w})$ at $37^{\circ} \mathrm{C}$ for $16 \mathrm{~h}$. Following digestion, the mixture was acidified by the addition of $10 \mu \mathrm{l}$ formic acid. Thirdly, the peptides were fractionated using strong cation exchange chromatography with a LC-20AB high performance liquid chromatography pump system (Shimadzu Corporation, Kyoto, Japan). Peptides were eluted at a flow rate of $1 \mathrm{ml} / \mathrm{min}$ with a gradient of buffer A for $10 \mathrm{~min}, 5-60 \%$ buffer B (25 mM NaH $\mathrm{PO}_{4}, 1 \mathrm{M} \mathrm{KCl}$ in $25 \% \mathrm{CAN}$; $\left.\mathrm{pH} 2.7\right)$ for $27 \mathrm{~min}$ and $60-100 \%$ buffer B for $1 \mathrm{~min}$ at $25^{\circ} \mathrm{C}$. The system was subsequently maintained at $100 \%$ buffer B for $1 \mathrm{~min}$ before equilibration with buffer $\mathrm{A}$ for $10 \mathrm{~min}$ prior to the next injection. Elution was monitored by measuring the absorbance at $214 \mathrm{~nm}$ and fractions were collected every $1 \mathrm{~min}$. The eluted peptides were pooled into 20 fractions, desalted with a Strata X C18 column (Phenomenex, Inc., Tianjin, China) and vacuum dried. Each fraction was subsequently resuspended in buffer $\mathrm{C}$ (5\% ACN, 0.1\% FA) and centrifuged at 20,000 $\mathrm{xg}$ for $10 \mathrm{~min}$ at $4^{\circ} \mathrm{C}$; the final concentration of peptide was approximately $0.5 \mu \mathrm{g} / \mu \mathrm{l}$. Supernatant $(10 \mu \mathrm{l})$ was loaded on a LC-20AD nanoHPLC (Shimadzu Corporation) by the autosampler onto a $2 \mathrm{~cm} \mathrm{C18} \mathrm{trap} \mathrm{column.} \mathrm{Peptides} \mathrm{were} \mathrm{eluted} \mathrm{onto} \mathrm{a} 10 \mathrm{~cm}$ analytical C18 column (inner diameter, $75 \mu \mathrm{m}$ ) packed in-house. The samples were loaded at $8 \mu \mathrm{l} / \mathrm{min}$ for $4 \mathrm{~min}$. The gradient was then run at $300 \mathrm{nl} / \mathrm{min}$ for $35 \mathrm{~min}$, starting from 2 to $35 \%$ buffer D (95\% ACN, 0.1\% FA), followed by a 5 min linear gradient to $60 \%$ and a 2 min linear gradient to $80 \% /$ Buffer D was maintained at $80 \%$ for $4 \mathrm{~min}$, and finally returned to $5 \%$ in $1 \mathrm{~min}$. Data acquisition was performed with a TripleTOF 5600 System (Shanghai AB SCIEX Analytical Instrument Trading Co., Shanghai, China) fitted with a Nanospray III source (Shanghai AB SCIEX Analytical Instrument Trading Co.) and a pulled quartz tip as the emitter (New Objective, Inc., Woburn, MA, USA). Data were acquired using a positive-ion spray voltage of $2.5 \mathrm{kV}$, curtain gas of $30 \mathrm{psi}$, nebulizer gas 
Table I. Clinical characteristics of the subjects from whom the AF, maternal plasma and VF samples were collected.

\begin{tabular}{|c|c|c|c|c|c|c|c|c|}
\hline & \multicolumn{2}{|c|}{$\mathrm{AF}$} & \multicolumn{2}{|c|}{ Plasma } & \multicolumn{2}{|c|}{$\mathrm{VF}$ of PROM } & \multicolumn{2}{|c|}{ VF of healthy control } \\
\hline & $<37$ weeks & $\geq 37$ weeks & $<37$ weeks & $\geq 37$ weeks & $<37$ weeks & $\geq 37$ weeks & $<37$ weeks & $\geq 37$ weeks \\
\hline $\begin{array}{l}\text { Maternal age, y } \\
\text { Mean } \pm \text { SD (range) }\end{array}$ & $\begin{array}{c}25.9 \pm 3.6 \\
(19-33)\end{array}$ & $\begin{array}{c}27.4 \pm 3.8 \\
(22-34)\end{array}$ & $\begin{array}{c}25.9 \pm 3.6 \\
(19-33)\end{array}$ & $\begin{array}{c}27.8 \pm 4.8 \\
(19-39)\end{array}$ & $\begin{array}{c}26.7 \pm 5.6 \\
(19-38)\end{array}$ & $\begin{array}{c}25.7 \pm 3.3 \\
(20-35)\end{array}$ & $\begin{array}{c}25.4 \pm 3.6 \\
(20-31)\end{array}$ & $\begin{array}{c}27.2 \pm 4.8 \\
(20-38)\end{array}$ \\
\hline $\begin{array}{l}\text { Gestational age at } \\
\text { sample collection, } \mathrm{d}\end{array}$ & $145.8 \pm 10.4$ & $276.7 \pm 6.2^{\mathrm{a}}$ & $145.8 \pm 10.4$ & $276.9 \pm 6.4^{\mathrm{a}}$ & $221.3 \pm 34.9$ & $274.8 \pm 6.7^{\mathrm{a}}$ & $227.6 \pm 39.3$ & $275.8 \pm 8.4$ \\
\hline Mean \pm SD (range) & $(127-163)$ & $(260-286)$ & $(127-163)$ & $(260-286)$ & $(174-258)$ & $(265-286)$ & $(147-258)$ & $(260-288)$ \\
\hline $\begin{array}{l}\text { Gravidity, } \mathrm{n} \\
\text { Mean } \pm \text { SD (range) }\end{array}$ & $\begin{array}{c}2.0 \pm 1.0 \\
(1-4)\end{array}$ & $\begin{array}{c}2.3 \pm 1.3 \\
(1-5)\end{array}$ & $\begin{array}{c}2.0 \pm 1.0 \\
(1-4)\end{array}$ & $\begin{array}{c}2.9 \pm 1.6 \\
(1-6)\end{array}$ & $\begin{array}{c}2.9 \pm 2.0 \\
(1-7)\end{array}$ & $\begin{array}{c}2.2 \pm 1.3 \\
(1-5)\end{array}$ & $\begin{array}{c}1.9 \pm 1.4 \\
(1-5)\end{array}$ & $\begin{array}{c}1.8 \pm 0.7 \\
(1-3)\end{array}$ \\
\hline Parity, $\mathrm{n}$ & $0.2 \pm 0.4$ & $0.4 \pm 0.5$ & $0.2 \pm 0.4$ & $0.6 \pm 0.7$ & $0.6 \pm 0.5$ & $0.3 \pm 0.5$ & $0.1 \pm 0.3$ & $0.4 \pm 0.5$ \\
\hline Mean \pm SD (range) & $(0-1)$ & $(0-1)$ & $(0-1)$ & $(0-2)$ & $(0-1)$ & $(0-1)$ & $(0-1)$ & $(0-1)$ \\
\hline
\end{tabular}

${ }^{a} \mathrm{P}<0.05,<37$ weeks vs. $\geq 37$ weeks pregnant women. AF, amniotic fluid; VF, vaginal fluid; PROM, premature rupture of membranes; $\mathrm{SD}$, standard deviation.

of $15 \mathrm{psi}$ and an interface heater temperature of $150^{\circ} \mathrm{C} . \mathrm{MS}$ analysis was performed in a high sensitivity scan mode. The MS scan range was $350-1,500 \mathrm{~m} / \mathrm{z}$. The top 30 precursor ions were selected into the $\mathrm{MS}^{2}$ scan, and the MS/MS scan range was $350-1,250 \mathrm{~m} / \mathrm{z}$. The data were analyzed using the Mascot search engine (Matrix Science, Ltd., London, UK; version 2.3.02) for protein identification. Protein function methods were described by the Gene Ontology (GO) system and the Cluster of Orthologous Groups of Proteins (COGs) database.

ELISA and Magnetic Luminex ${ }^{\circledR}$ screening assay. A total of 14 proteins were detected. Sandwich ELISA kits purchased from RayBiotech, Inc. (Norcross, GA, USA) and Cloud-Clone Corp. (Houston, TX, USA) were used to analyze AF and maternal plasma samples for the concentrations of EGF-containing fibulin-like extracellular matrix protein 1 (EFEMP1; cat. no. SEF422Hu), keratin type II cytoskeletal 4 (KRT4; cat. no. SEA489Hu), keratin type II cytoskeletal 6A (KRT6A; cat. no. SED234Hu), keratin type II cytoskeletal 8 (KRT8; cat. no. SEC025Hu), keratin type I cytoskeletal 15 (KRT15; cat. no. SEA517Hu), keratin type I cytoskeletal 17 (KRT17; cat. no. SEB822Hu), keratin type I cytoskeletal 19 (KRT19; cat. no. ELH-CYT19-1), BPI fold-containing family A member 1 (BPIFA1 or PLUNC; cat. no. ELH-PLUNC-1), pulmonary surfactant-associated protein B (SFTPB; cat. no. SEB622Hu) and zymogen granule protein 16 homolog B (ZG16B; cat. no. SES158Hu). The assays were performed according to the manufacturers' protocols. Dilutions (100 $\mu \mathrm{l}$ each) of standard, blank and diluted samples was added into a 96 well plate in duplicate. Plates were incubated for $2.5 \mathrm{~h}$ at room temperature (RT) followed with gentle for $2 \mathrm{~h}$ at $37^{\circ} \mathrm{C}$ Following washing, biotinylated antibodies were incubated for $1 \mathrm{~h}$ at RT with gentle shaking at $37^{\circ} \mathrm{C}$. Horseradish peroxidase-stretavidin solution was incubated for $45 \mathrm{~min}$ at RT followed by gentle shaking for $30 \mathrm{~min}$ at $37^{\circ} \mathrm{C}$. Tetramethylbenzidine dihydrochloride substrates were added into each well for $30 \mathrm{~min}$ in the dark. The reactions were terminated by adding $0.2 \mathrm{M}$ sulfuric acid. The absorbance of each well was recorded at a wavelength of $450 \mathrm{~nm}$.
The concentrations of insulin-like growth factor-binding protein 2 (IGFBP2), mesothelin, placental protein 14 (PP14), and serpin family B member 3 (serpin B3) in AF, maternal plasma and VF samples were determined by Magnetic Luminex ${ }^{\circledR}$ Screening Assay multiplex kits (R\&D Systems, Inc., Minneapolis, MN, USA). The kits were used according to the manufacturer's protocol. The microparticle cocktail $(50 \mu \mathrm{l})$ was added into a 90 well plate, followed by $50 \mu \mathrm{l}$ standard and diluted samples. The microplates were incubated for $2 \mathrm{~h}$ at RT with gentle shaking. A volume of $50 \mu \mathrm{l}$ diluted biotin antibody cocktail and diluted streptavidin-phycoerythin were added and incubated at RT with shaking. The microparticles were re-suspended with $100 \mu \mathrm{l}$ wash buffer. Following incubation for $2 \mathrm{~min}$, the microplates were read using the Luminex ${ }^{\circledR}$ Liquid Chip.

Lateral flow assay development. A lateral flow assay based on colloidal gold immunochromatography technology was developed to qualitatively detect PP14. A concentration of $1 \mathrm{mg} / \mathrm{ml}$ mouse monoclonal to anti-pp14 antibody (cat. no. ab17247; Abcam, Cambridge, UK) was conjugated with $40 \mathrm{~nm}$ colloidal gold particles, and the antibody-gold conjugate was atomized into a glass fiber pad with an AirJet dispenser (BioDot, Inc., Irvine, CA, USA). A concentration of $1 \mathrm{mg} / \mathrm{ml} \mathrm{rabbit} \mathrm{anti-pp14}$ polyclonal antibody (cat. no. abs124712; Absin, Shanghai, China) and $0.5 \mathrm{mg} / \mathrm{ml}$ goat-anti-mouse immunoglobulin $\mathrm{G}$ (cat. no. TA130072; OriGene Technologies, Inc., Beijing, China) were atomized as a test line and control line (BioDot, Inc.), respectively. Recombinant human PP14 protein (Abcam) was diluted to $0.04,0.02,0.01,0.008,0.005$ and $0.004 \mu \mathrm{g} / \mathrm{ml}$ with $0.01 \mathrm{M}$ PBS. Diluted samples $(80 \mu \mathrm{l})$ were dropped into the sample wells of the lateral flow strip, and the results were subsequently observed within $10 \mathrm{~min}$. A positive result was judged when the test line and the control line appeared. A negative result was determined when only the control line was visible.

Statistical analysis. The normality of distribution of continuous variables was tested by a one-sample Kolmogorov-Smirnov 
test in SPSS version 13.0 for Windows (SPSS Inc., Chicago, IL, USA). Continuous variables with a normal distribution are presented as the mean \pm standard deviation; non-normal variables were reported as median (interquartile range). The means of two and three or more groups of continuous normally distributed variables were compared by independent sample Student's t-test or one-way analysis of variance (ANOVA), respectively. If the ANOVA was significant, Holm-Sidak's post-hoc test was used to analyze the difference between two groups. The Mann-Whitney U-test or Kruskal-Wallis test was used to compare the means of two and three or more groups of variables not normally distributed, and Dunn's post hoc test was performed to determine the difference between two groups if the P-value of the Kruskal-Wallis test was $<0.05$. The diagnostic values of each detected protein were evaluated via Receiver Operating Characteristic (ROC) curve and the area under the curve (AUC). Statistical analysis was performed using GraphPad Prism 7 (GraphPad Software Inc., La Jolla, CA, USA). $\mathrm{P}<0.05$ was considered to indicate a statistically significant difference.

\section{Results}

Protein identification by $L C-M S / M S$. Two pooled samples were analyzed by LC-MS/MS. A total of 648,074 identified spectra [1.09\% false discovery rate (FDR)] and 625,162 identified spectra $(0.89 \%$ FDR) were obtained in AF and maternal plasma samples, respectively. 4,343 peptides and 896 proteins were identified in AF samples, and 3,788 peptides and 681 proteins were identified in maternal plasma samples.

COGs analysis illustrated 21 classifications of proteins in $\mathrm{AF}$ and maternal plasma samples. For AF samples, proteins focused on the functional class $\mathrm{O}$ (post-translation modification, protein turnover and chaperones), class $\mathrm{R}$ (general function prediction) and class $\mathrm{G}$ (carbohydrate transport and metabolism). However, for maternal plasma samples, a greater number of proteins focused on Class O, Class R and Class Z (cytoskeleton) (data not shown).

GO analysis demonstrated that there was similar functional grouping and protein localization for proteins in $\mathrm{AF}$ and maternal plasma. However, the proteins in AF have a unique molecular function called protein tag, compared with proteins in maternal plasma (data not shown).

Unique proteins in AF sample. Subsequent to eliminating proteins identified in $\mathrm{AF}$ and maternal plasma, 540 unique proteins were observed for AF (data not shown). COG functional classification revealed that these proteins were involved in post-translational modification, protein turnover, chaperones, general function prediction, carbohydrate transport and metabolism. The GO system analysis demonstrated that these proteins were involved in 'development', 'reproduction, single-organism processes', 'organelle' and 'binding' (data not shown). Proteins EFEMP1, KRT4, KRT6A, KRT8, KRT15, KRT17, KRT19, PLUNC, SFTPB, ZG16B, IGFBP2, mesothelin, PP14 and serpin B3 were selected for further evaluation. Criteria for their selection is presented in Fig. 1.

Expression levels of 14 proteins in $A F$ and maternal plasma. Expression levels of the 14 proteins in small panel samples

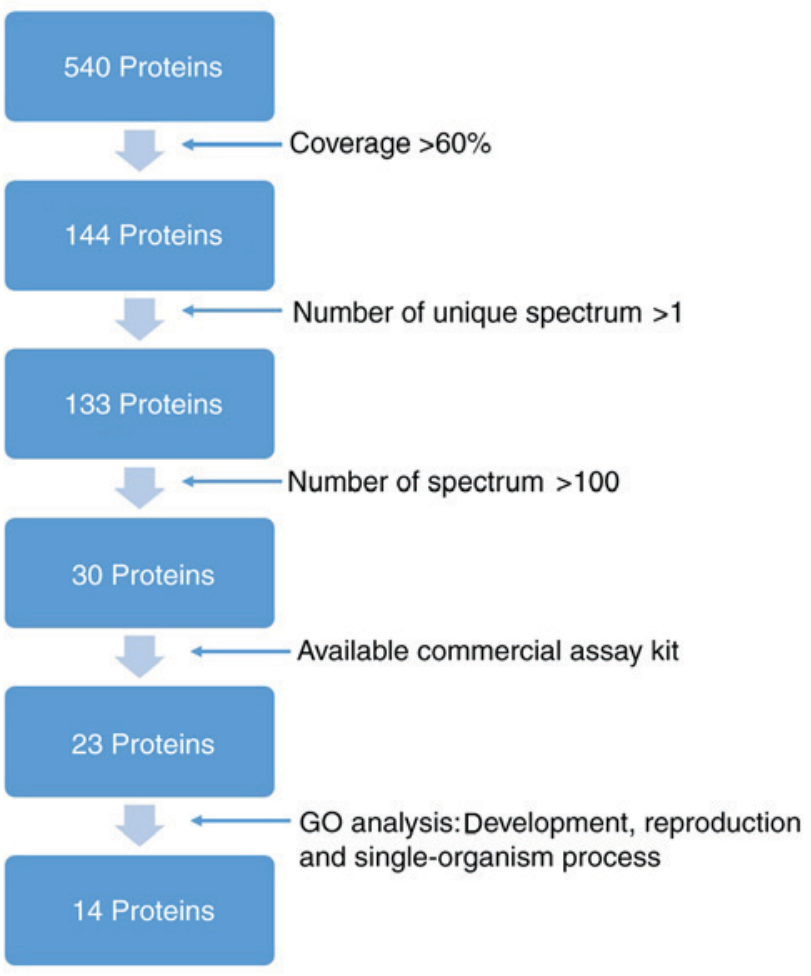

Figure 1. Criteria of selecting proteins for further detection.

were detected initially. The results demonstrated that the expression levels of KRT6A, KRT8, KRT15 and KRT17 in maternal plasma samples were higher compared with in AF samples, and thus these proteins were excluded for further analysis (data not shown). The expression levels of SFTPB, PLUNC, ZG16B, EFEMP1, KRT4 and KRT19 were detected by ELISA (Fig. 2). Additionally, the expression levels of PP14, IGFBP2, mesothelin and serpin B3 were determined (Fig. 2) using a magnetic Luminex ${ }^{\circledR}$ screening assay for the $\mathrm{AF}$ and maternal plasma samples. The expression levels of SFTPB and PLUNC in AF significantly decreased, and PP14 expression levels significantly increased during the second trimester, compared with those of the third trimester $(\mathrm{P}<0.05)$. In maternal plasma samples, there was no detection of KRT19 via ELISA. The ELISA kit detected SFTPB in 28 maternal plasma samples ( 8 from the second trimester and 20 from the third trimester). For EFEMP1, the expression levels of 18 maternal plasma samples from the second trimester and 95 maternal plasma samples from the third trimester were undetected. As the number of samples that were detected was insufficient, for the purpose of the statistical analysis, the undetected samples were assigned a value with the minimum corresponding detectable levels stated by the ELISA kit. The results revealed that no significant differences were observed between the second and third trimester groups in the expression levels of all proteins in the maternal plasma samples $(\mathrm{P}>0.05)$. For SFTPB, the expression levels in AF during the second trimester were not statistically significant different from the maternal plasma of the second trimester $(\mathrm{P}>0.05)$.

Expression levels of KRT19, PP14, IGFBP2, mesothelin and serpin $B 3$ in $V F$ samples. As the expression level of SFTPB in $\mathrm{AF}$ of the second trimester was not significantly different 

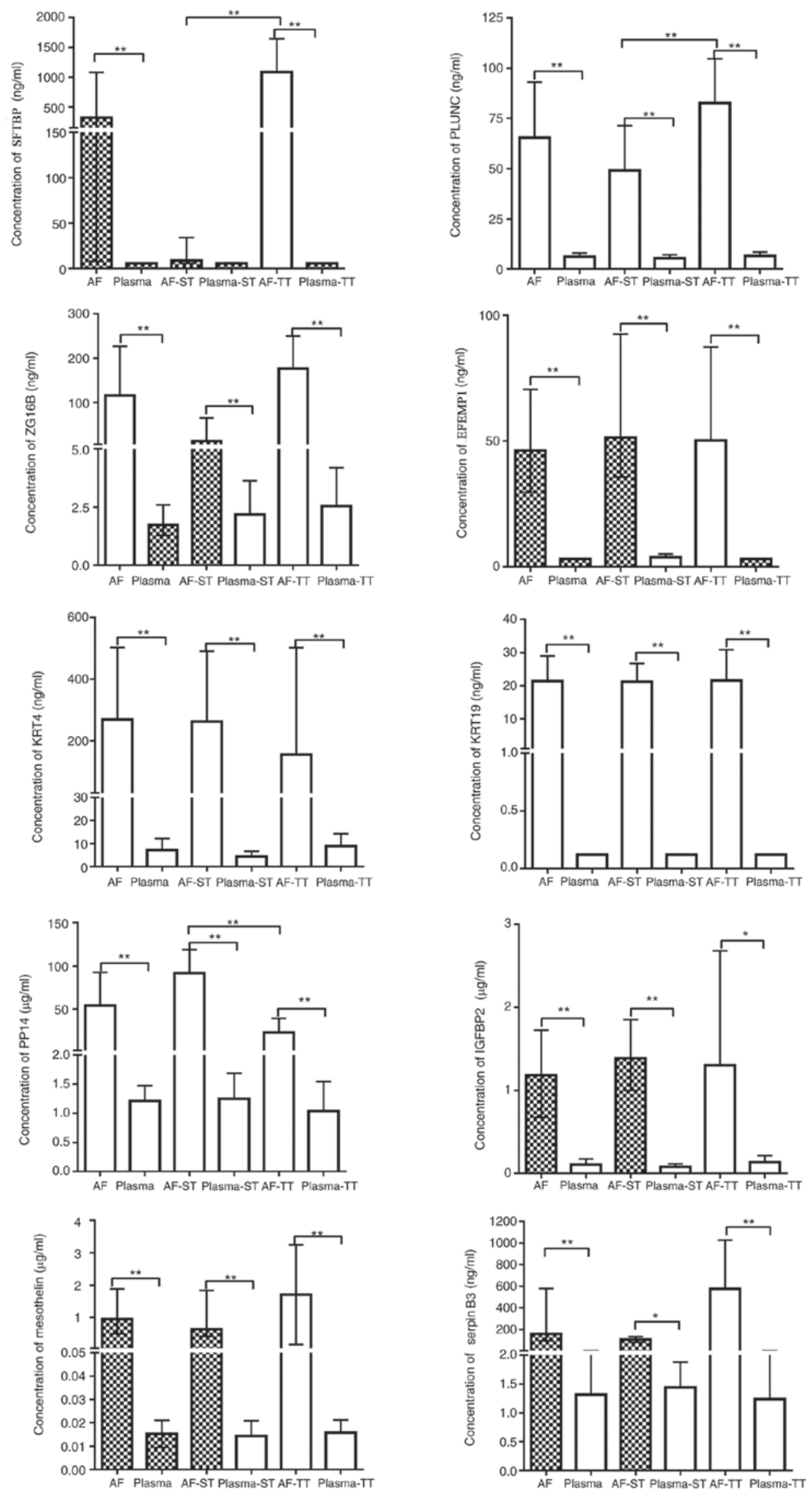

Figure 2. Concentrations of SFTPB, PLUNC, ZG16B, EFEMP1, KRT4, KRT19, PP14, IGFBP2, mesothelin and serpin B3 in AF and maternal plasma. Blank bars mean continuous variables are normally distributed. Filled bars mean continuous variables are non-normally distributed. The normal variables are presented as the mean \pm standard deviation (above error bar); the non-normal variable error bars are presented as the median (interquartile range). $\mathrm{P}<0.05$, ${ }^{* *} \mathrm{P}<0.001 . \mathrm{SFTPB}$, pulmonary surfactant-associated protein B; PLUNC, BPI fold-containing family A member 1; ZG16B, zymogen granule protein 16 homolog B; EFEMP1, EGF-containing fibulin-like extracellular matrix protein 1; KRT4, keratin, type II cytoskeletal 4; KRT19, keratin, type I cytoskeletal 19; PP14, placental protein 14; IGFB2, insulin-like growth factor-binding protein 2; serpin B3, serpin family B member 3; AF, amniotic fluid; ST, second trimester; TT, third trimester. 

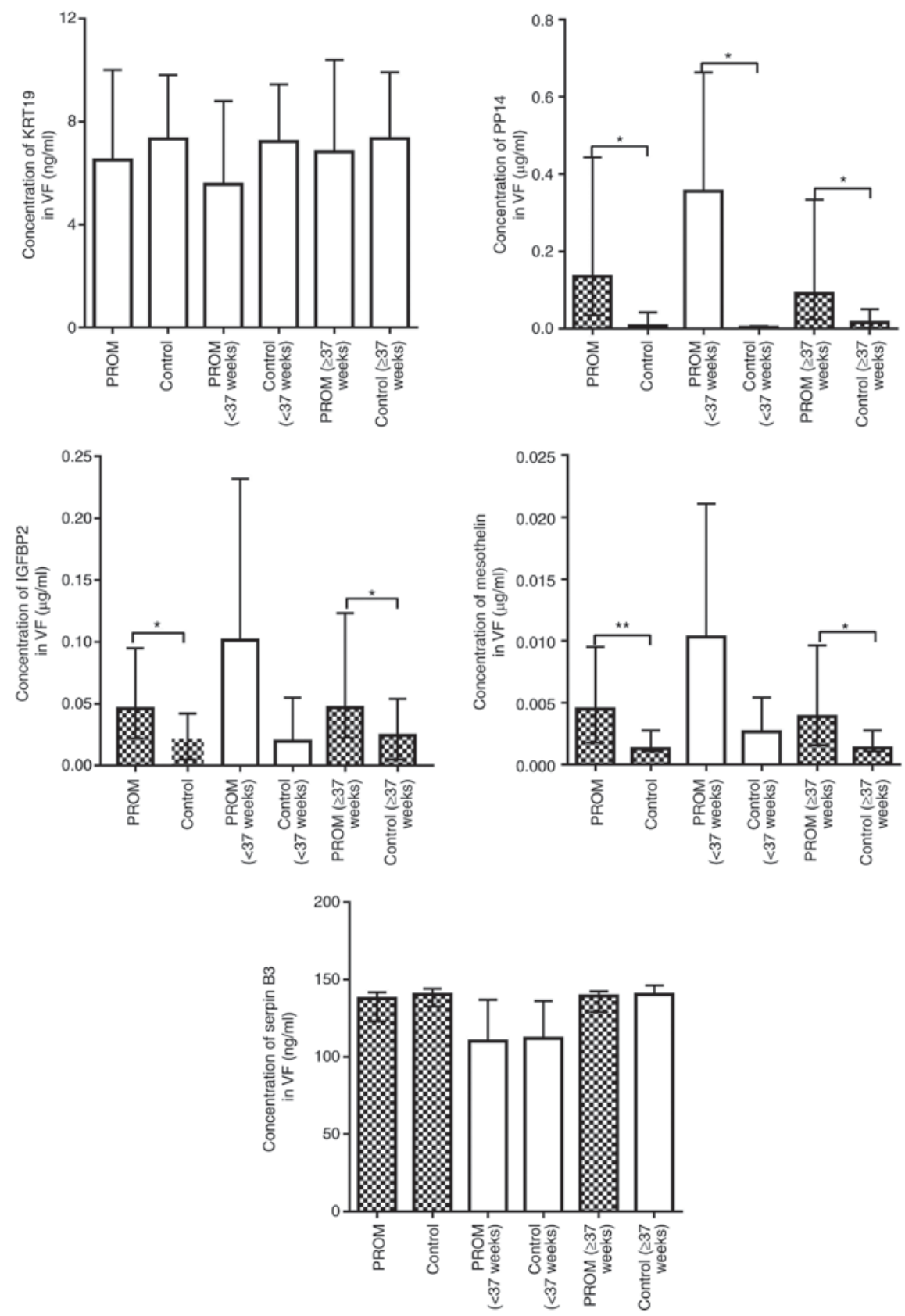

Figure 3. Concentrations of KRT19, PP14, IGFBP2, mesothelin and serpin B3 in the VF of women with overall PROM, PROM ( $<37$ weeks) and PROM ( $\geq 37$ weeks) and overall control, control ( $<37$ weeks) and control ( $\geq 37$ weeks). Blank bars mean continuous variables are normally distributed. Filled bars mean continuous variables are non-normally distributed. The normal variables are presented as the mean \pm standard deviation (above error bar); the normal variables are presented as the median (interquartile range). ${ }^{*} \mathrm{P}<0.05,{ }^{* *} \mathrm{P}<0.001$. KRT19, keratin, type I cytoskeletal 19; PP14, placental protein 14; IGFBP2, insulin-like growth factor-binding protein 2; serpin B3, serpin family B member 3; VF, vaginal fluid; PROM, premature rupture of membranes.

compared with that in maternal plasma in the same trimester, PLUNC, ZG16B, EFEMP1, KRT4, KRT19, PP14, IGFBP2, mesothelin and serpin B3 were considered more valuable for evaluating VF samples. Unfortunately, since the expression levels of the majority of samples were below the minimum detectable limit of the ELISA kit, no valid data were obtained for the expression levels of PLUNC, ZG16B, EFEMP1 and KRT4 in VF samples. The results (Fig. 3) demonstrated that the expression levels of KRT19 and serpin B3 were not significantly different in the overall PROM groups compared with the control groups, PROM for $\geq 37$ weeks vs. control for $\geq 37$ weeks, or PPROM for $<37$ weeks vs. control for $<37$ weeks $(\mathrm{P}>0.05)$. Expression levels of mesothelin and
IGFBP2 in the PROM group were significantly increased compared with those in the control group $(\mathrm{P}<0.05)$. However, following $<37$ weeks and $\geq 37$ weeks classification, the expression levels of mesothelin and IGFBP2 in the PPROM groups were not significantly different compared with those in the control for $<37$ weeks $(\mathrm{P}>0.05)$, but were significantly different at $\geq 37$ weeks compared with the control group $(\mathrm{P}<0.05)$. Therefore, mesothelin and IGFBP2 were observed to be more suitable for PROM diagnosis, occurring at $\geq 37$ weeks of pregnancy. As for PP14, the expression level was significantly increased in the PROM group compared with the control group at either $<37$ weeks or $\geq 37$ weeks $(\mathrm{P}<0.05)$. 
To investigate whether the expression levels of mesothelin, IGFBP2 and PP14 in VF samples may have been altered due to blood contamination, analysis of the data in blood-contaminated and blood-free VF samples was conducted (Table II). The results indicated that the expression levels of mesothelin, IGFBP2 and PP14 in VF samples of the PROM (either $<37$ weeks or $\geq 37$ weeks) or control ( $\geq 37$ weeks) groups was not significantly different in blood-contaminated samples compared with blood-free samples $(\mathrm{P}>0.05)$. There was only one blood-contaminated VF sample in control ( $<37$ weeks), thus the data are not presented. These results indicated that mesothelin, IGFBP2, and PP14 may be potential biomarkers for diagnosing PROM.

Diagnostic value of mesothelin, IGFBP2, and PP14 for PROM. The ROC curve was used to evaluate the diagnostic values of mesothelin, IGFBP2 and PP14 for PROM (Fig. 4). PP14 was observed to have an excellent diagnostic accuracy for PPROM, with a respective sensitivity and specificity of 100 and $87.5 \%$ for a cutoff value of $0.008 \mu \mathrm{g} / \mathrm{ml}$ (AUC of $0.99 \pm 0.02, \mathrm{P}<0.001$ ).

PP14-based lateral flow assay. The human recombinant PP14 protein was used to determine the visible threshold of the PP14 strip. Different concentrations (0.04, 0.02, 0.01, 0.008, 0.005 and $0.004 \mu \mathrm{g} / \mathrm{ml}$ ) of PP14 diluted with $0.01 \mathrm{M} \mathrm{PBS}$ were added to the test strip, and detection was performed three times for each sample. The results demonstrated that the PP14-based strip may be effective in clinical use and had a detection threshold of $0.008 \mu \mathrm{g} / \mathrm{ml}$ (Fig. 5).

\section{Discussion}

In a previous study, a biomarker [soluble intercellular adhesion molecule-1 (sICAM-1)] was identified for the diagnosis of PROM, using a cytokine antibody array (9). A rapid test strip for clinical use was developed, based on colloidal gold immunochromatography technology. The validity of the strip was $95 \%$ (10). The product was approved for access to the medical market by China's SFDA. However, a limitation of the product is that the results may be false positive if the VF is contaminated with blood. Therefore, an aim of the present study was to discover novel biomarkers that are not influenced by blood contamination using LC-MS/MS-based proteomic techniques.

As an excellent biomarker for diagnosing PROM, sICAM-1 was observed to have high expression in AF and low expression in VF with an intact fetal membrane throughout the whole pregnancy. Once PROM occurred, AF was disseminated into the vagina and diluted by VF; sICAM-1 was observed to have high detectable expression levels in VF of PROM, regardless of the existence of blood. AF contains numerous proteins; certain proteins are derived from maternal blood and others from the fetus (11-13). Proteins derived from the fetus are more suitable PROM biomarkers. Therefore, a number of proteins were selected that primarily have functions associated with development or reproduction, for further quantitative detection and evaluation of diagnostic value.

In the present study, 14 unique proteins in $\mathrm{AF}$ were examined according to the selection criteria. The results demonstrated that the expression levels of these 14 unique 

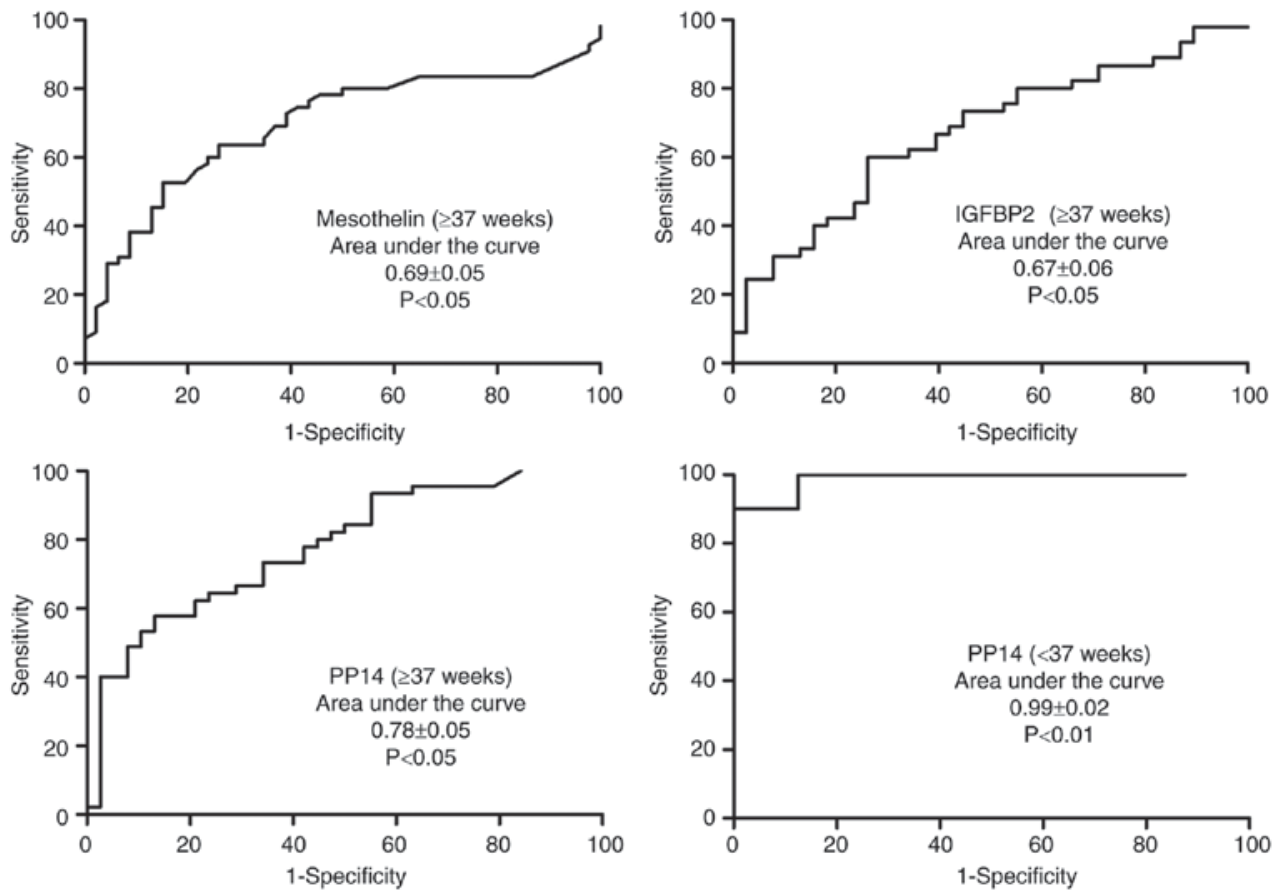

Figure 4. Receiver operating characteristic curves of mesothelin, IGFBP2 and PP14 for diagnosing PROM. IGFBP2, insulin-like growth factor-binding protein 2; PP14, placental protein 14; PROM, premature rupture of membranes.

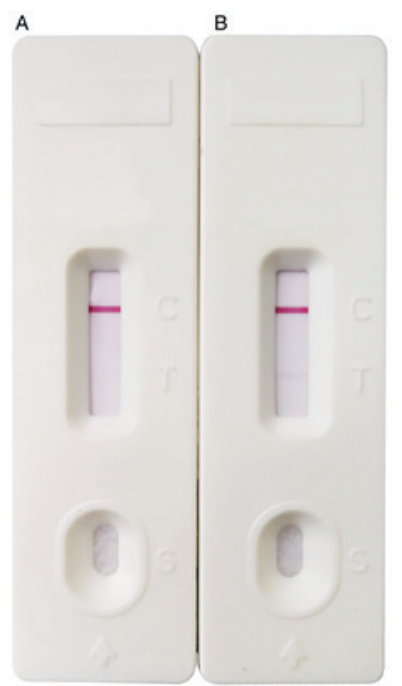

Figure 5. Results of PP14-based lateral flow assay. (A) A total of $0.005 \mu \mathrm{g} / \mathrm{ml}$ PP14 recombinant protein was added. The strip was negative. (B) A total of $0.008 \mu \mathrm{g} / \mathrm{ml} \mathrm{PP} 14$ recombinant protein was added. The strip was positive. PP14, placental protein 14; C, control line; T, test line; S, sample well.

proteins could be detected in AF samples and maternal plasma samples, with KRT6A, KRT8, KRT15 and KRT17 exhibiting higher expression levels in maternal plasma samples compared with AF samples, which appeared to contradict the results of the LC-MS/MS analysis. This may be explained by the characteristics of ELISA. It known that false positive signals from ELISA may be caused by heterophilic antibodies, which in the case of the present study were the human anti-mouse antibodies in the blood (14). The intensity of the signal is easily influenced by ambient temperature, as higher temperatures are able to increase the optical density value. The quality of the ELISA kit may additionally interfere with the results.

The present results demonstrated that mesothelin, IGFBP2 and PP14 had potential value for diagnosing PROM. Mesothelin is a 40-kDa membrane-glycoprotein; it is reported to associate with a number of types of cancer, although, its biological function in normal conditions is unclear (15). IGFBP2 is a $36-\mathrm{kDa}$ protein, which is primarily involved in metabolic disease and cancer (16). PP14 is a secretory glycoprotein produced by the endometrium during pregnancy and has a high expression level in AF (17). The results of the present study demonstrated that mesothelin and IGFBP2 had insufficient ability to diagnose PPROM, and PP14 was the superior biomarker for diagnosing PPROM, with a respective sensitivity and specificity of 100 and $87.5 \%$ with a cutoff value of $0.008 \mu \mathrm{g} / \mathrm{ml}$. The present study revealed that the expression level of PP14 in AF during the second trimester was significantly higher compared with in the third trimester. This was in agreement with previous studies that the concentration of PP14 in AF reached its peak at 18-20 weeks of gestation, and subsequently decreased $(18,19)$. This may explain why PP14 had a higher accuracy in diagnosing PPROM compared with PROM ( $\geq 37$ weeks). Furthermore, the concentration of PP14 in VF samples was stable in blood-contaminated VF and blood-free VF samples, which is an advantage for the future clinical use of PP14.

As the cutoff value of PP14 for diagnosis of PPROM was at the level of $\mu \mathrm{g} / \mathrm{ml}$, a colloid-gold lateral flow strip was developed to rapidly test PP14. The strip was revealed to have a detection threshold of $0.008 \mu \mathrm{g} / \mathrm{ml}$, which was in accordance with the cutoff value of PP14. This strip may become a useful and rapid tool to supply indicatory information for diagnosing PPROM in hospitals, particularly in vaginal bleeding-complicated cases. 
However, there were five limitations to the present study. First, no sufficient volume of VF samples for LC-MS/MS analysis was available. As $50 \mathrm{ml} \mathrm{VF}$ samples was required, it was impossible to obtain the proteome profile of $\mathrm{VF}$ samples. Second, a total of 540 unique proteins were identified in AF samples. However, it was impossible to examine all of them. Accordingly, only 14 proteins of interest were selected for confirmation analysis, and thus it is possible that some effective biomarkers were missed. Third, the sample size of PPROM was small. A greater number of samples are required for further verification that PP14 may be an excellent biomarker for PPROM, which is not influenced by blood contamination. Fourth, the accuracy of the PP14 strip for clinical samples of PPROM requires confirmation using large samples, and multiple-center and single-blind clinical trials. As a standard clinical trial must be approved by China SFDA, this may be a future direction. Fifth, the expression levels of STFBP, KRT19 and EFEMP1 in maternal plasma samples, and PLUNC, ZG16B, EFEMP1 and KRT4 levels in VF samples were undetectable, so accurate levels could not be determined. This may have been caused by insufficient ELISA kit sensitivity.

To the best of the authors' knowledge, the present study was the first study to provide data on the expression levels of SFTPB, PLUNC, ZG16B, EFEMP1, KRT4, KRT19, PP14, IGFBP2, mesothelin and serpin B3 in AF and maternal plasma during the second and third trimester of pregnancy. Expression levels of KRT19, PP14, IGFBP2, mesothelin and serpin B3 in PROM and non-PROM VF samples in the Chinese population may aid the understanding of physiological processes experienced by pregnant women. Finally, the present study suggested that PP14 may be a novel potential biomarker for PPROM and that the PP14-based strip may be a helpful bedside test for rapidly diagnosing PPROM.

\section{Acknowledgements}

Not applicable.

\section{Funding}

The present study was supported by grants from the National Natural Science Foundation of China (grant no. 81501261), the Sichuan Provincial Science \& Technology Project (grant nos. 2016SZ0013 and 2015SZ0054-2) and the Office of Science \& Technology of Chengdu (grant no. 2015-HM01-00431-SF).

\section{Availability of data and materials}

The datasets used and/or analyzed during the current study are available from the corresponding author on reasonable request.

\section{Authors' contributions}

YW made substantial contributions to conception and design of the study. HL made substantial contributions to data acquisition and was involved in drafting the manuscript. GC, BZ, LG and TW analyzed and interpreted the data. YL, JG and QY collected samples and critically revised the manuscript for important intellectual content. YuL was accountable for all aspects of the work, in ensuring that questions related to the accuracy or integrity of the research were appropriately investigated and resolved, and analyzed the data. LZ made substantial contributions to conception and design of the study, data acquisition, revised the manuscript critically for important intellectual content, and gave final approval of the version to be published. All authors read and approved the final manuscript.

\section{Ethics approval and consent to participate}

The present study was approved by the ethics committees of West China Second University Hospital of Sichuan University (Sichuan, China) and Shuangliu District Maternal and Child Health Hospital (Sichuan, China). All subjects signed consent forms.

\section{Consent for publication}

All patients provided written informed consent for the publication of any associated data and accompanying images.

\section{Competing interests}

The authors declare that they have no competing interests.

\section{References}

1. Mariona FG and Cabero L: Are we ready for a new look at the diagnosis of premature rupture of membranes? J Matern Fetal Neonatal Med 25: 403-407, 2012.

2. Jiang HL, Wang $X$ and Zhang WY: Investigation of premature rupture of membrane in Beijing area. Chin J Clin 74-76, 2015 (In Chinese).

3. Medina TM and Hill DA: Preterm premature rupture of membranes: Diagnosis and management. Am Fam Physician 73: 659-664, 2006.

4. Waters TP and Mercer B: Preterm PROM: Prediction, prevention, principles. Clin Obstet Gynecol 54: 307-312, 2011.

5. El-Messidi A and Cameron A: Diagnosis of premature rupture of membranes: Inspiration from the past and insights for the future. J Obstet Gynaecol Can 32: 561-569, 2010.

6. Lee SM, Lee J, Seong HS, Lee SE, Park JS, Romero R and Yoon BH: The clinical significance of a positive Amnisure test in women with term labor with intact membranes. J Matern Fetal Neonatal Med 22: 305-310, 2009.

7. Liang DK, Qi HB, Luo X, Xiao XQ and Jia XY: Comparative study of placental $\alpha$-microglobulin-1, insulin-like growth factor binding protein-1 and nitrazine test to diagnose premature rupture of membranes: A randomized controlled trial. J Obstet Gynaecol Res 40: 1555-1560, 2014.

8. Abdelazim IA, Abdelrazak KM, Al-Kadi M, Yehia AH and Abdulkareem AF: Fetal fibronectin (Quick Check fFN test) versus placental alpha microglobulin-1 (AmniSure test) for detection of premature rupture of fetal membranes. Arch Gynecol Obstet 290: 457-464, 2014.

9. Wang T, Zhou R, Zhang L, Wang Y, Song CP, Lin W, Niu X, Lin Y and $\mathrm{Hu} \mathrm{H}$ : Proteins in leaked amniotic fluid as biomarkers diagnostic for prelabor rupture of membranes. Proteomics Clin Appl 5: 415-421, 2011.

10. Wang T, Zhou R, Xiong W, Wang Y, Zhu C, Song C, Gao L, Zhang L and $\mathrm{Hu} \mathrm{H}$ : Clinical evaluation of soluble intercellular adhesion molecule-1 and insulin like growth factor-binding protein-1-based rapid immunoassays for the diagnosis of prelabor rupture of membranes. J Perinat Med 41: 181-185, 2013.

11. Tong XL, Wang L, Gao TB, Qin YG, Qi YQ and Xu YP: Potential function of amniotic fluid in fetal development-novel insights by comparing the composition of human amniotic fluid with umbilical cord and maternal serum at mid and late gestation. J Chin Med Assoc 72: 368-373, 2009. 
12. Chen WW: Studies on the origin of human amniotic fluid cells by immunofluorescent staining of keratin filaments. J Med Genet 19: 433-436, 1982.

13. Tydén O, Bergström S and Nilsson BA: Origin of amniotic fluid cells in mid-trimester pregnancies. Br J Obstet Gynaecol 88: 278-286, 1981.

14. Grebenchtchikov N, Sweep CG, Geurts-Moespot A, Piffanelli A, Foekens JA and Benraad TJ: An ELISA avoiding interference by heterophilic antibodies in the measurement of components of the plasminogen activation system in blood. J Immunol Methods 268: 219-231, 2002.

15. Baldo P and Cecco S: Amatuximab and novel agents targeting mesothelin for solid tumors. Onco Targets Ther 10: 5337-5353, 2017.

16. Russo VC, Azar WJ, Yau SW, Sabin MA and Werther GA: IGFBP-2: The dark horse in metabolism and cancer. Cytokine Growth Factor Rev 26: 329-346, 2015.
17. Rachmilewitz J, Riely GJ, Huang JH, Chen A and Tykocinski ML: A rheostatic mechanism for T-cell inhibition based on elevation of activation thresholds. Blood 98: 3727-3732, 2001.

18. Bischof P: Three pregnancy proteins (PP12, PP14, and PAPP-A) Their biological and clinical relevance. Am J Perinatol 6: 110-116, 1989.

19. Chatzakis K, Wathen N, Campbell J, Iles R, Dawnay A and Chard T: Dramatic increase in levels of placental protein 14 in amniotic fluid at 10-15 weeks' pregnancy. Early Hum Dev 36: 113-116, 1994.

This work is licensed under a Creative Commons Attribution-NonCommercial-NoDerivatives 4.0 International (CC BY-NC-ND 4.0) License. 